\title{
Neurofibroma of Nasal Cavity and Nasopharynx
}

\author{
Mahendra I Katre ${ }^{1 *}$, Sanjay Sonawle ${ }^{2}$ and Sunil Deshmukh ${ }^{1}$ \\ ${ }^{1}$ Department of ENT and Head Neck Oncology Government cancer Hospital, India \\ ${ }^{2}$ Department of ENT BJMedical College, India
}

*Corresponding author: Dr. Mahendra I Katre, Assistant Professor Department of ENT and Head Neck Oncology , Government cancer Hospital Aurangabad, Maharashtra University of Health Science Nashik, India

Submission: 侮 September 18, 2017; Published: 海 December 08, 2017

\begin{abstract}
Neurofibroma may occur in any parts of myelinated nerves having Schwann cells. However it is extremely rare in the nasal septum. We have had experience with an isolated neurofibroma of the nasal septum which was successfully removed by trans-nasal endoscopic excision. This article aimed to describe the clinical, endoscopic surgical and pathological features of this unusual tumor of nasopharynx.

Keywords: Neurofibroma; Myelinated nerve; Nasal septum
\end{abstract}

\section{Introduction}

Neurofibroma is a neurogenic tumour arising from Schwann cells or peripheral tissues of nerve sheaths. It is usually presented with Von Recklinghausen's disease rather than as a solitary tumour [1]. It is commonly found in the head and neck and the flexoral surfaces of the upper and lower extremities. In the head-and-neck region, it often originates from the vestibular nerve. However, neurofibroma arising from the nose and paranasal sinuses are rare, and especially those from the nasal septum are extremely rare [1].

The treatment of choice for neurofibroma in the nose and paranasal sinuses is complete excision via a lateral rhinotomy approach or a mid-facial degloving approach [1,2]. However, if neurofibroma is present as a small solitary tumour, it is curable by adequate primary excision [1]. Recently, the technique of endoscopic nasal surgery has rapidly developed, and transansal endoscopic excision of the primary lesion is successful when neurofibroma is a solitary tumour present in nose and paranasal sinus.

\section{Case Report}

A 17-year-old male patient presented to department of ENT and Head Neck OPD with a history of progressive bilateral nasal obstruction, persistent nasal discharge since 1 year, headache and hyposmia over the past 3 month. He had neither history of epistaxis, pain, abnormal skin pigmentation nor a family history of neural tumour. Diagnostic nasal endoscopy revealed a firm, huge mass completely filling the bilateral posterior choana .The mass had a smooth mucosal surface, pale in color, was ovoid in shape. On diagnostic endoscopy we found that mass was attached to posterior edge of nasal septum and the postero-superior portion of the right side nasal septum. Nasopharyngeal roof was normal.

\section{Oral cavity}

Soft palate was bulged, Uvula is centrally located with normal movement

- $\quad$ Rest ENT examination Within normal limit

- $\quad$ No lymph node palpable, no neurological deficit .

- Ophthalmic examination: Within normal limit.

\section{CT PNS}

A CT scan with contrast-enhancement demonstrated a large soft-tissue density mass in the right nasal cavity, not very well enhancing extending in right choana and nasopharynx. The septum had a deviation to the left side as a result of the mass in the right nasal cavity. Rest of sinuses were normal (Figure 1).

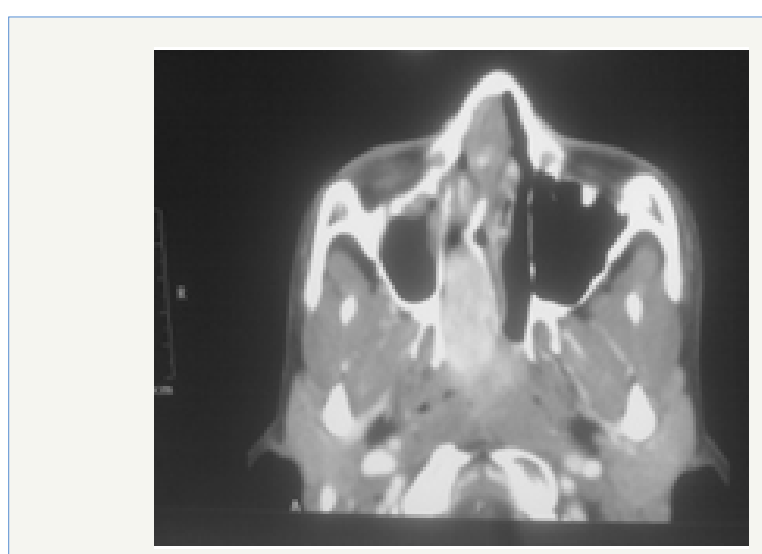

Figure 1: Coronal cuts. mass in it nasal cavity and Nasopharynx. 


\section{Surgical Excision}

The plan of endoscopic excision of mass under general anesthesia was made. Mucosal decongestion achieved with nasal pattis and 1:10000 (4\% Xylocaine and adrenalin solution). Previous finding of endoscopy confirmed. Mass was attached to posterior edge of nasal septum and the postero-superior portion of the right side nasal septum. Incision taken on posterior bony portion of right side of septum. Septal flap elevated on right side over bony septum, bony attachment of mass cauterized with bipolar coutery and mass delivered into per orally. Hemostasis achieved and nasal packing done. The resected mass was not encapsulated but relatively circumscribed. The cut surface of the mass was pale, gray-white, solid and homogeneous (Figure 2). Histological examination of the mass reviled Encapsulated mass showed combined proliferation of eosinophilic, thin, wavy collagen fibres lying in a disorderly pattern and spindle-shaped cells with a wavy, serpentine configuration and pointed ends and the spindle-shaped tumour cells.

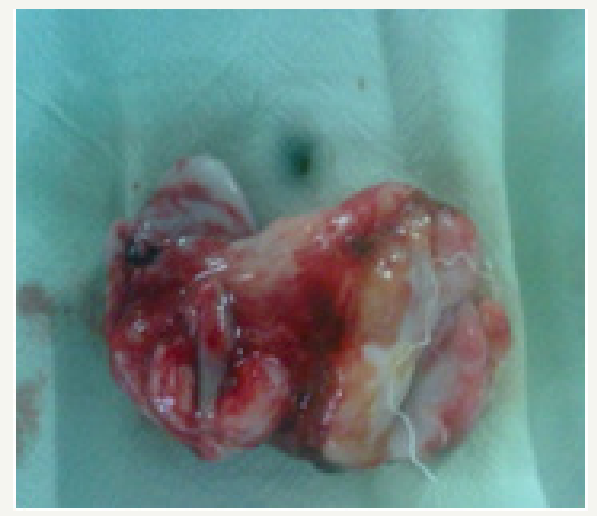

Figure 2: In to Specimen of nasopharyngeal mass.

The stroma was infiltrated with lymphocytes and mast cells. Mitoses were not observed. The final pathological diagnosis made was neurofibroma arising from the right nasal septum. After transnasal endoscopic surgical removal, no other surgical treatments have been performed. The post-operative course was uneventful. And no recurrence of neurofibroma has been observed during 12-month follow-up.

\section{Discussion}

Peripheral nerve-sheath tumors are divided into neurofibroma, schwannoma and neurogenic sarcoma. Neurofibroma and schwannoma are classified as benign, and neurogenic sarcoma as malignant [1]. All peripheral nerve-sheath tumors are believed to arise from Schwann cells. They often arise from sensory nerves and are found in the head and neck and the flexoral surface of the upper and lower extremities. However, they are rarely found in the nasal cavity and paranasal sinuses. In the nasal cavity and paranasal sinuses, the great majority of the peripheral nerve sheath tumors reported were schwannom as. A review of 430,000 pathological specimens by revealed six neurofibroma involving the nasal cavity or paranasal sinuses [3]. Almost all neurofibromas are associated with Von Recklinghausen's disease, so isolated neurofibromas without this association are extremely rare. Isolated peripheral nerve-sheath tumors occur commonly in females, in the fifth and sixth decades [4].

The neurofibroma occurring in the nose may be solitary or multiple. If neurofibromas are plexiform they would be often part of neurofibromatosis type-I. In the nose and paranasal sinuses, the tumour arises from the first and second division of the trigeminal nerve and from autonomic plexuses but cannot arise from the olfactory nerve, which has no Schwann cells [1]. A combined nasoethmoid involvement is most common. It is followed, in order of frequency, by maxillary sinus, nasal cavity and sphenoid sinus [1]. In the nasal septum, it is extremely rare, and nearly all have been solitary tumors. Clinically, symptoms and signs are dependent on the site. Usually common symptoms and signs are nasal obstruction, epistaxis, facial pain, swelling and proptosis. Schwannoma often produce a painful sensation and tenderness and are predominantly centrifugally distributed, whereas neurofibromas are asymptomatic and primarily centripetally located. The diagnosis of neurofibroma is usually made only when the lesion is biopsied, because of the non-specific nature of these symptoms and signs [5].

Histologically, the neurofibroma is non-encapsulated, poorly circumscribed with an ill-defined margin. Tumour is normal smooth surface slow growing and generally asymptomatic. It may develop at any age and there is no gender predilection $[6,7]$. This is typified by relatively hypocellular proliferation of bland, palely eosinophilic spindle cells with rather wavy, S-shaped or buckled nuclei set in a copious fibrillary or rather myxoid background. Usually, mitoses are not seen. Small nerve fibers are usually readily identified within the tumour. The stroma of these lesions occasionally undergoes marked myxoid or hyaline change. Ultra structurally, this is composed of an admixture of Schwann cells and perineural fibroblasts, and these cells are S-100 positive $[3,8]$. In contrast, a schwannoma is a truly encapsulated tumour and shows two distinct patterns, Antoni-A and Antoni-B. The nuclei are arranged in pallisades with space between the rows, forming the so-called Verocay bodies.

The treatment of choice for benign peripheral nerve-sheath tumors is total local excision. Although neurofibroma is more invasive and more likely to recur after removal than schwannoma, recurrence is rare. Complete local excision is usually curative [1,2] and prognosis is excellent, if the tumour is completely removed. Malignant transformation of neurofibroma is rare unless the patient has neurofibromatosis $[1,7]$. In those situations malignant degeneration about $10 \%$ to occurs in $15 \%$ of the time [9].

\section{Conclusion}

Neurofibroma is a peripheral nerve-sheath tumour. Solitary neurofibroma of the nasal septum and nasopharynx is extremely rare, and curable by complete local excision via a transnasal endoscopic approach.

\section{References}

1. Batsakis JG (1979) Tumours of the Head and Neck Clinical and Pathological considerations. (Eds.), Tumours of the Head and Neck 
Clinical and Pathological considerations $\left(2^{\text {nd }}\right)$ Williams, Wilkins Baltimore, in Maryland,pp. 573.

2. Stevens DJ, Kirkham N (1988) Neurofibroma of paranasal sinuses. J larygology otol 102: 256-259.

3. Perzin K, panyu $H$ (1982) nonepithelial tumours of the nasal cavity ,paranasal sinuses and nasopharynx Cancer 50:2193-2202.

4. Morris JH (1989) The nervous system. In: Cotran RS, Robbin's Pathologic Basis of Disease. WB Saunders, Philadelphia, pp. 1445-1446.

5. Yong DK, Chang HB, Jang SSk, WonS (1997) Rhinology 35: 89-91.
6. Campora DE, Radii M, Campora DL (1999) Neurogenic tumours of head and neck in children .Int J pediatr otolaryngol 49 suppl 1: S231-233.

7. Glimer Hill H.S, Kline DG (2000) Neurogenic tumours of cervical vagus nerve report of 4 cases and review of literature. Neurosurgery 46(6): 1498-1503.

8. Agarwal MK (1979) Neurofibroma of the maxillary antrum. Oral Surg Oral Pathol 48(2): 150-152.

9. Devita VT, Hellman S (1989) Cancer Principles and practice of oncology. In: Chang AE, Rosenberg SA, et al. (Eds.) Sarcomas of Soft Tissue. J.B. Lippincott Company,(6 $6^{\text {th }}$ edn), Williams \& Wilkins ,Philadelphia, in Pennsylvania, pp. 1345-1398. 